\title{
Normalising Brain PET Images
}

Elia Ferrando Juliá, Daniel Ruiz Fernández, and Antonio Soriano Payá

Department of Computer Technology. University of Alicante, PO 99, 03080 Alicante, SPAIN

\{eferrando, druiz, soriano\}@dtic.ua.es

Key words: PET, brain, normalize, 3D images

\begin{abstract}
PET is a nuclear medical examination which constructs a three-dimensional image of metabolism inside the body; in this article in particular, images are taken from the brain. The high complexity inherent to the interpretation of the brain images makes that any help is important to the specialists in order to accurate the diagnostic. In order to reach reliable and good images, a normalization process is suggested in this paper, consisting of centring the brain in the three-dimensional image, scaling it according to a template brain and, finally, rotating the brain according to the inclination of the template. For not reducing the quality of the information the application works with PET image format and radioactivity measures instead of translate to an ordinary colour image.
\end{abstract}

\section{Introduction}

In the last years the development of medical diagnosis task has been excellent. One of the improvements has been the use of advanced technological devices in diagnostic tasks. Specifically in medical image we find a great evolution from X-Ray to Positron Emission Tomography, doing necessary the use of computer algorithms to get better images because a clear image can be essential for doing an exact diagnostic.

Positron Emission Tomography (PET) is a technique based on the detection of radioactivity from a certain part of a living body. The radiation is caused by the emission of positrons emitted from a kind of fluorescent glucose injected to the bloodstream of the patient who has been fasting for at least 6 hours, and who has a suitably low blood sugar [1].

The tomography helps to visualize the biochemical changes that take place inside a body, like metabolism. The main advantage of this technique compare to other nuclear medicine tests, is that PET detects metabolism within body tissues, whereas other types just detect the amount of a radioactive substance collected in body tissue to examine the tissue's function [2]. The usually procedure is to administrate fluorodeoxyglucose ${ }^{1}$ to the patients which is a kind of sugar for fitting brain cells. Specifically it is used ${ }^{18}$ F-FDG for assessment its

${ }^{1}$ Its chemical formula is $\mathrm{C}_{6} \mathrm{H}_{11} \mathrm{FO}_{5}$ and its chemical name is 2-Fluoro-2-Deoxy-DGlucose abbreviated by FDG. 
glucose metabolism that has 109.8 minutes of half-life. The glucose is retained by cells with high metabolic activity, on the contrary, tissues with low or nonactivity means damage regions [3]. These radioactive nuclei decays by emitting positrons that annihilate with electrons at the tissue. When a positron crashes against an electron, their collision produces two gamma rays having the same energy, but going in 180 directions. The gamma rays leave the patient's body and are detected by the PET scanner [4]. The specialized scanner emits light when it is hit by nuclear radiation from the patient, which is emitted from the ultra violet to the infrared range of wavelength $(100-800 \mathrm{~nm})$. Then the amount of data of radioactivity is collect to feed into a computer to be converted into a complex picture. There are several image formats that a scanner can make, nevertheless we have been working with ECAT 7 file format.

PET analysis is commonly used in oncology [5] because malignant tumours are identified by tissues with high metabolic activity as in neurological field [6] because high radioactivity is associated with brain activity and it helps to diagnose illnesses such as epilepsy, stroke or dementia. Besides, in cardiology [7], PET images are used for study atherosclerosis and vascular disease and finally, in pharmacology [8] it is used to test new drugs injected to animals.

The reason of working with radioactivity concentration instead of translate data image to a standard ${ }^{2}$ colour image format is due to keep up all information about metabolism patient to be analyzed with accuracy. If translation to a standard image format is carried out, we can lost radioactivity information.

\section{Objectives and Methods}

The main objective of this work is to supply tools to prepare PET images to be analyzed using an automatic decision support system. The application has been made to process ranges of radioactivity from ECAT 7 files instead of some arbitrary colours [9]. These kinds of images are structured in three parts (Fig. 1):

1. Main header. It has information about the file, such as file type, patient's data, isotope name, isotope half-life, etc. In order to work with the files and to comply with the law of data protection, we deleted the patient name keeping his privacy.

2. Subheader. It stores specific information of the image matrix, for instants, $\mathrm{X}, \mathrm{Y}$ and $\mathrm{Z}$ dimensions, data type, size of each edge of a voxel from each dimension, etc.

3. Image data. It is a three-dimensional array in which each cell contains the real quantitative value of the radioactivity detected in each point of the brain and the assessment unit is $\mathrm{Bq} / \mathrm{ml}^{3}$ or $\mathrm{kBq} / \mathrm{ml}^{4}$, which are the radioactivity

\footnotetext{
${ }^{2}$ TIFF, JPEG, GIFF or similar.

${ }^{3}$ Bequerel(s)/millilitre.

${ }^{4}$ Kilobequerel(s)/millilitre.
} 


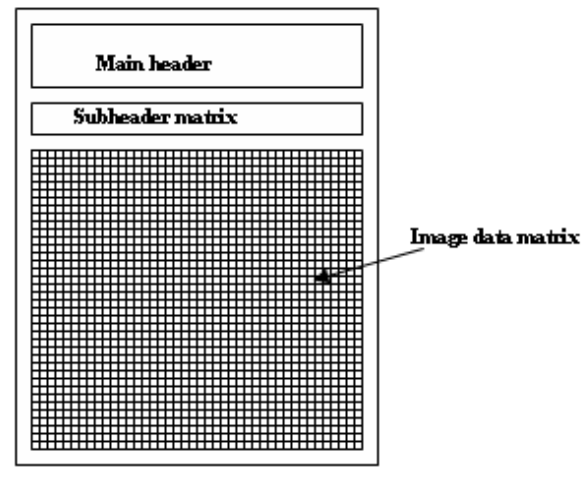

Fig. 1. Structure of an ECAT file.

concentration per the volume of the voxel ${ }^{5}$. There is one three-dimensional array for one frame of time, however, we just work with static images. One horizontal slice of a brain is stored in a two-dimensional array (Fig. 2).

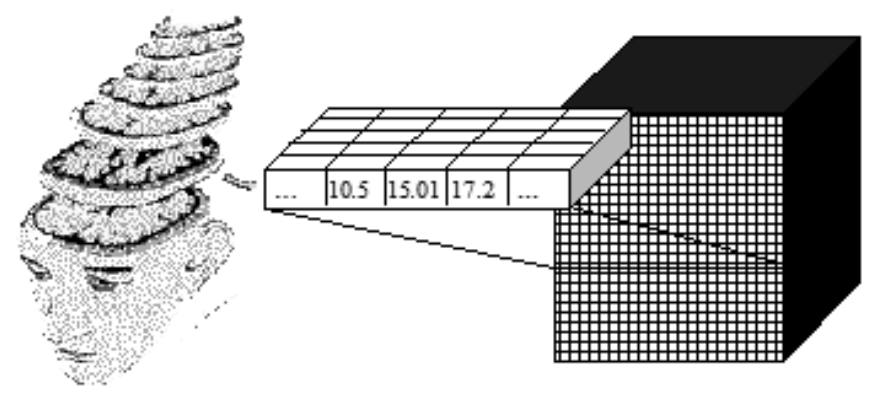

Fig. 2. The structure of PET raw data stored in the matrix image.

When the patient is inside of a scanner, he has some possibility of movement, so he could nodded his head, additionally the most likely is that patient brain has different size comparing to template brain, hence we need to normalize the PET images to be compare between them and classify in health or damage brains.

To analyze PET images is necessary to have a model of reference to take from it the normalization parameters, like the degrees of inclination or the value of the area at the middle horizontal plane. In this work, we have a real human brain from which we know perfectly the areas, the inclination and its health estate. Normalization process could not be in other way, because all volume brains are different each one from another, so it is not possible to associate

\footnotetext{
${ }^{5}$ A cell represents a voxel when the image is printed.
} 
to a geometrical figure or something like that. This work is centred to correct three main characteristics: position of the brain in the image, the scale and the inclination.

\section{Normalization Process}

Every kind of image taken from a situation like a photograph, a TAC or a PET, all of them have noise. Then, the first step is to eliminate this noise using a filter. We identify noise with negative and very low values of radioactivity because it represents non-meaningful information.

It is also interesting to segment an image leaving just the brain and taking out cover tissues, because depending on the scan process it can take more or less regions out of the brain and the centre and volume could be unreliable [10]. So we have distributed radiation values in seven ranges, like rainbow colours because it is the best way to assign levels of metabolism (Fig. 3 Left). We recognized first and second level such cover tissues, which are not useful for medical test, consequently we just take into account the rest of levels (Fig. 3 Right).
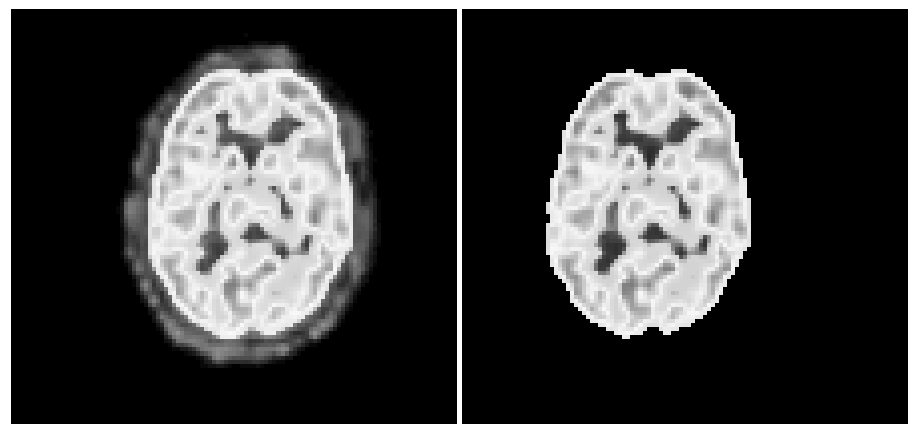

Fig. 3. (Left) Brain distributed in seven levels of radioactivity. (Right) Segmented brain.

\subsection{Centring the Brain}

To compare a PET image with the template, it is necessary to situate the brain at the centre of the image (CM) (Fig. 4). In order to do this operation we calculate the centre of mass (Eq. 1) of the brain and move it to the middle of three dimensions of the image.

$$
C M=\frac{\left(\sum x_{i}+\sum y_{i}+\sum z_{i}\right)}{v}
$$

where $\mathrm{x}_{i}, \mathrm{y}_{i}$ and $\mathrm{z}_{i}$ are voxel coordinates of the brain segmented and $\mathrm{v}$ is the volume of the entire brain segmented. CM obtained is the centre of the brain in 
the non-normalized image, therefore we have to move the brain until the middle of image and this is done adding the distance difference calculate in the next expressions:

$$
M o v_{x}=\frac{\operatorname{dim}_{x}}{2}-1-x_{i} ; \operatorname{Mov}_{y}=\frac{\operatorname{dim}_{y}}{2}-1-y_{i} ; M o v_{z}=\frac{\operatorname{dim}_{z}}{2}-1-z_{i}
$$

where $\operatorname{dim}_{x}, \operatorname{dim}_{y}$ and $\operatorname{dim}_{z}$ are values of dimension in $\mathrm{X}$ axis, $\mathrm{Y}$ axis and $\mathrm{Z}$ axis respectively and we subtract a unit because the image matrix data is numbered from 0 to dimension-1.

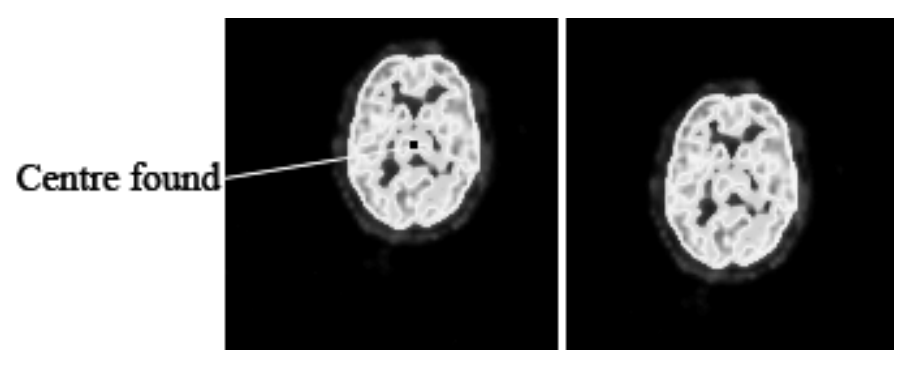

Fig. 4. (Left) Brain non-centred. (Right) Brain centred.

\subsection{Scaling the Brain}

Next function to use in normalization process is scaling the brain volume (Fig. 5 . We have to estimate how bigger or smaller is the non-normalized image than the template and this is done using the area of the central horizontal plane of the PET image (Eq. 3).

$$
p=\sqrt{\frac{\text { areaImage }}{\text { areaTemplate }}}
$$

The value $\mathrm{p}$ obtained is the proportion to apply to each coordinate of the matrix image.

$$
x_{f}=x_{i} \cdot p \quad y_{f}=y_{i} \cdot p \quad z_{f}=z_{i} \cdot p
$$

The $\mathrm{x}_{f}, \mathrm{y}_{f}$ and $\mathrm{z}_{f}$ obtained are destination coordinates and $\mathrm{x}_{i}, \mathrm{y}_{i}$ and $\mathrm{z}_{i}$ are source coordinates. If $\mathrm{p}$ is more than a unit, we use interpolation (average of the neighbours values) to fill the empty values of the new voxels added due to the increase of the image. On the contrary, if $\mathrm{p}$ is less than a unit, interpolation is not necessary to be applied to the image because there is not empty voxels.

\subsection{Rotating the Brain}

Finally, the brain must be at the same degrees inclination than the template brain. Our idea is based on to the comparison of the outline brains (Fig. 6). So, 

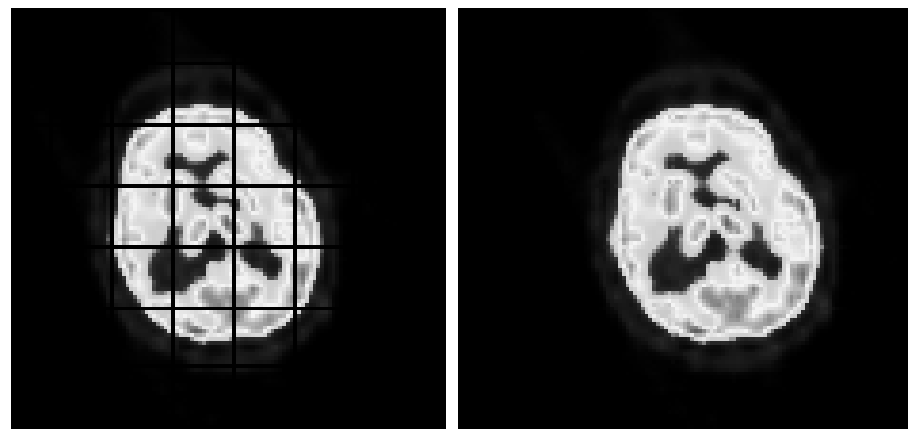

Fig. 5. (Left) Initial scale image. (Right) Final scale image.

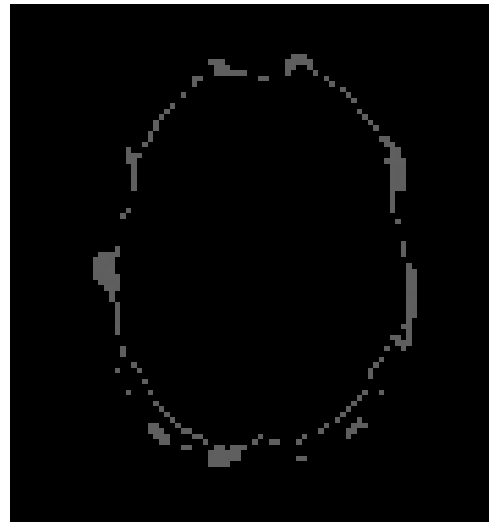

Fig. 6. Outline of the template brain.

we implement a function to extract outline of a brain which is made up filtering third range of radiation frequency.

The rotation process has been approached from several points of view. The first one was oriented to find the maximum radius from template and from the patient brain. It works looking for the voxel which was situated at the highest distance. Then, the patient brain was rotated the degrees difference between the maximum radius of the template and from the patient. We discard this method because all brains are not identically, they have different outlines hence the maximum radius found was not the same for all the brains.

Secondly, we draw a rectangle rounding the brain in the middle planes at every dimensions and check tangent points between template and patient brain, after that, patient image was rotated the degrees difference. This mechanism was discarded due to the same problem as the previous method.

Finally we use the outline coordinates lists of the patient and template brain image, making up two mathematical series to analyze the correlation between them. We have used the coefficient of Pearson because it measures the correla- 


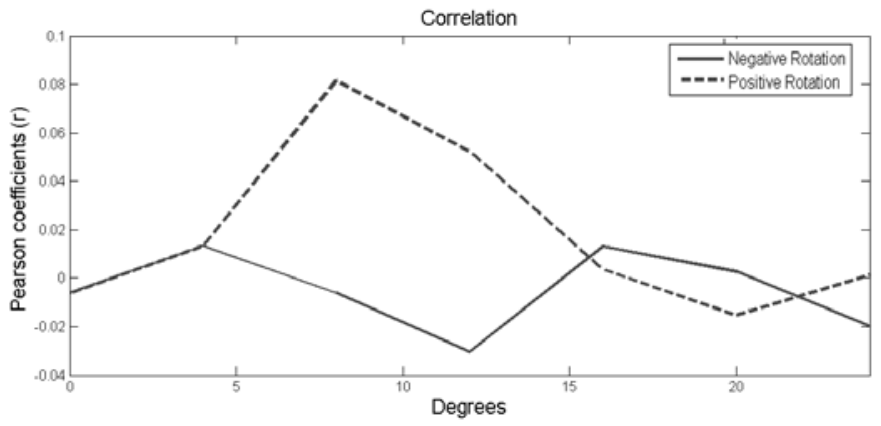

Fig. 7. Pearson coefficients of correlation: (Left) Negative rotation, (Right) Positive rotation.

tion between two random linear series (Fig. 7), looking for their tendency to increase or decrease together [11]. It was already implemented by open source. The method implemented follows these instructions:

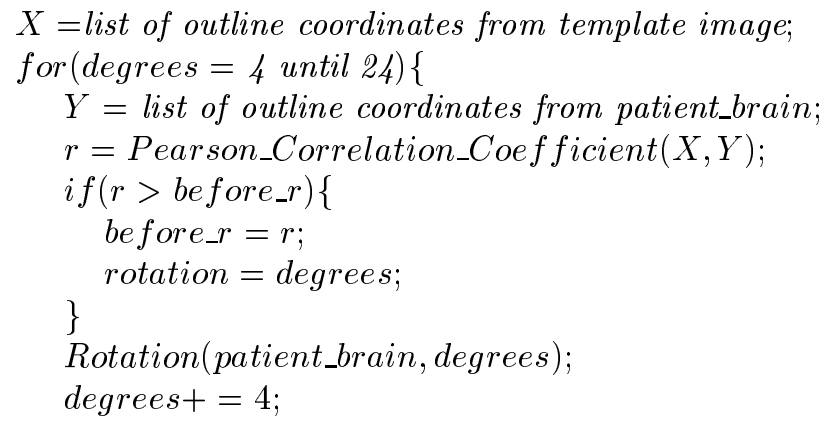

It is the same mechanism to rotate to opposite direction excepting that degrees are negative and when the process is finished, it rotate the patient image the degrees reached where the correlation coefficient was the biggest. This same process is executes for the three dimensions. The implementation to rotation coordinates is based on trigonometric functions (Eq. 5) where is moved each voxel to the position at degrees calculated before.

$$
\begin{gathered}
x_{f}=x_{i} \cdot \cos (\text { degrees })-y_{i} \cdot \sin (\text { degrees }) \\
y_{f}=x_{i} \cdot \sin (\text { degrees })+y_{i} \cdot \cos (\text { degrees })
\end{gathered}
$$

The $\mathrm{x}_{f}$ and $\mathrm{y}_{f}$ obtained are destination coordinates to where are going to move the source coordinates, $\mathrm{x}_{i}$ and $\mathrm{y}_{i}$. In the construction of the image rotate, as we are not working in a discreet scope, there are some differents source coordinates which are rotate to the same coordinates destined, this is a problem because it leaves some empty voxels in the rotate image (Fig. 8 Left). For that reason, rotation method has a second phase in which it renders the rotate image looking for empty voxels. When a empty voxel is found, it is calculated the coordinate to 
the back position in the original image, that mean, to rotate negatively and the empty voxel takes the value of the voxel in the coordinate calculated at original image (Fig. 8 Right)[12].
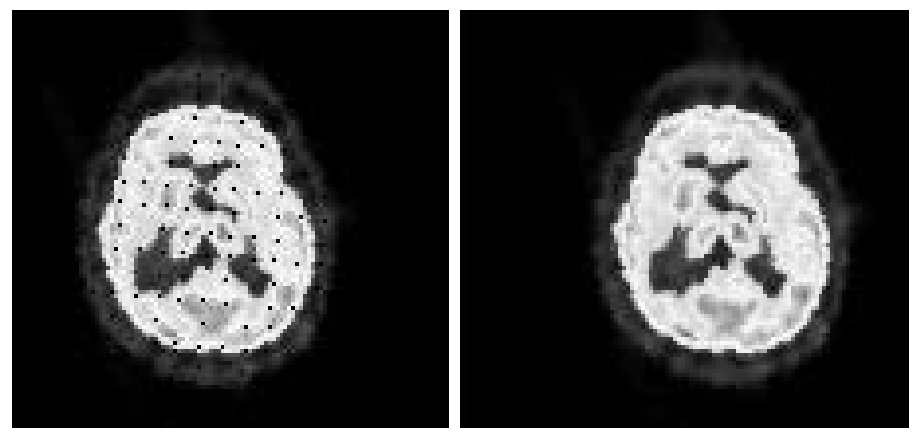

Fig. 8. (Left) Initial rotate image. (Right) Final rotate image.

\section{Conclusions}

We have implemented a set of tools which can do a preprocess of a PET image of the brain without lost of quality. This is possible because we operate always with the levels of radioactivity without convert the image to a graphics standard format. We use operations like centering, scaling and rotation to get an image that we can compare it with a template. Previously we have filtered the PET image eliminating noise and useless information.

We present this work as the previous one necessary to analise a PET image of the brain. Using the tools we have developed, nowadays we are implementing a diagnostic tool based in PET images to help radiologists and neurologists in the hard task of the diagnosis of brain diseases.

Acknowledgement. We appreciate the collaboration of Vesa Oikonen from the Turku PET Center helping us with the ECAT format and the libraries to read it. We are also grateful to Pedro Gonzalez Cabezas from PET Iberoa for lending us the PET images we have worked with.

\section{References}

1. Bailey, D.L., Townsend, D.W., Valk, P.E., Maisey, M.N.: Positron Emission Tomography. Publisher Springer (2005).

2. Silverman, D.H.S., Small, G.W., Chang, C.Y., Lu, C.S., Kung de Aburto, M.A., Chen, W., Czernin, J., Rapoport, S.I., Pietrini, P., Alexander, G.E., Schapiro, M.B., Jagust, W.J., Hoffman, J.M., Welsh-Bohmer, K.A., Alavi, A., Clark, C.M., 
Salmon, E., de Leon, M.J., Mielke, R., Cummings, J.L., Kowell, A.P., Gambhir, S.S., Hoh, C.K., Phelps, M.E.: Positron Emission Tomography in Evaluation of Dementia. Regional Brain Metabolism and Long-term Outcome. The Journal of the American Medical Association, Vol 286, 17 (2001).

3. Feng, D., Ho, D., Chen, K., Wu, L., Wang, J., Liu, R., Yeh, S.: An evaluation of the algorithms for determining local cerebral metabolic rates of glucose using positron emission tomography dynamic data. IEEE Xplore, 14 (1995) 697-710.

4. Camborde, M.L., Thompson, C.J., Togane, D.: A positron-decay triggered transmission source for positron emission tomography. IEEE Transactions on Nuclear Science, 51 (2004) 53-58.

5. Bengel, F.M., Ziegler, S.I., Avril, N., Weber, W., Laubenbacher, C., Schwaiger, M.: Whole-body positron emission tomography in clinical oncology, comparison between attenuation-corrected and uncorrected images. European Journal of Nuclear Medicine and Molecular Imaging, (1997) 1091-1098

6. Tai, Y.F., Piccini, P.: Applications of Positron Emission Tomography in Neurology. Journal of Neurology, Neurosurgery and Psychiatry, 75 (2004) 669-676

7. Johnson, T.R.C., Becker, C.R., Wintersperger, B.J., Herzog, P., Lenhard, M.S., Reiser, M.F.: Detection of Cardiac Metastasis by Positron-Emission TomographyComputed Tomography. Circulation 112 (2005) 61-62.

8. Cunningham, V.J., Gunn, R.N., Matthews, J.C.: Quantification in positron emission tomography for research in pharmacology and drug development. Nuclear Medicine Communications, 25 (2004) 643-646.

9. Bockisch, A., Beyer, T., Antoch, G., Freudenberg, L.S., Kuhl, H, Debatin, J.F., Muller, S.P.: Positron Emission Tomography/Computed Tomography-Imaging Protocols, Artifacts and Pitfalls. Molecular Imaging and Biology, 6 (2004) 188199.

10. Buchert, R., Wilke, F., Chakrabarti, B., Martin, B., Brenner, W., Mester, J., Clausen, M.: Adjusted Scaling of FDG Positron Emission Tomography Images for Statistical Evaluation in Patients with Suspected Alzheimer's Disease. Journal of Neuroimaging, 15 (2005) 348-355.

11. Allen, E.: An introduction to linear regression and correlation. WH Freeman (1976)

12. Semmlow, J.L.: Biosignal and Biomedical Image Processing, MATLAB-Based Applications. Marcel Dekker Ltd. 22 (2004) 